\section{P2-S3.13 EFFECTIVE HIV AND AIDS PREVENTION: IS FACTORS AFFECTING THE SPREAD IMPORTANT?}

doi:10.1136/sextrans-2011-050108.332

${ }^{1} \mathrm{M}$ Bankole, ${ }^{2} \mathrm{M}$ Bankole, ${ }^{3} \mathrm{~A}$ Adebowale, ${ }^{4} \mathrm{G}$ Iboma, ${ }^{5} \mathrm{O}$ Olonire. ${ }^{1}$ Ministry of Health Lagos, Alausa-Ikeja, Nigeria; ${ }^{2}$ Nigerian Institute of Medical Research, Yaba, Nigeria; ${ }^{3}$ University of Lagos, Idi-araba, Nigeria; ${ }^{4}$ Ministry of Health Lagos State, Alausa-Ikeja, Nigeria; ${ }^{5}$ Ministry of Health, Alausa-Ikeja, Nigeria

Background There is no doubt that the last two decades had witnessed a rising trend in the Global efforts at combating HIV and AIDs, more especially through the various treatment measures. However, though a lot had been achieved in the treatment of infected persons, the long and short term side effects of the drugs; patient drug adherence and follow-up issues are often problematic. Therefore, the most appropriate way to address the menace will be rigorous efforts geared towards adopting simple and effective prevention methods the world over. In developing countries such as found in Africa, including Nigeria, there are many factors militating against achieving a good success in reducing the spread of HIV and AIDs. The identified factors include: culture and tradition of the people, peer group influence, large family burden (Economy), marriage and religion. Hence, this work was undertaken to identify the most common factors contributing to the spread of HIV and AIDS in order to adopt adequate, practical and preventive solutions to tackle the identified factors in Lagos, Nigeria.

Method Questionnaires were distributed to 500 people in different locations in Lagos State, including one tertiary institution, 50 hair dressing and barbing salons, five community groups, 50 patent medicine dealers' shops, National Union of Road Transport Workers and ten randomly selected secondary schools in the five geographical zones in Lagos State between October 2009 and May 2010.

Result Out of the 500 people given questionnaires, only 350 (70\%) responded. Out of the 350 respondents, 315 (90\%) of them have had sex at one time or another, of which a highly significant $(\mathrm{p}>0.05)$ 157 (49.8\%) people have had unprotected sex in the past. Of this group, 70 (44.6\%) were sex with multiple partners while 87 (55.4\%) were sex with single partners. Fifty (31.9\%) people in this group adduced their reason for unprotected sex to marriage obligation, 20 $(12.7 \%)$ to religion, $30(19.1 \%)$ to culture and tradition, $40(25.5 \%)$ to peer influence while 37 (23.6\%) were due to economic reasons. Conclusion There is a large pool of people who are still engaged in unprotected sex especially with multiple sexual partners in Lagos, Nigeria. The reasons include social, economic, religious, marital and religious factors. Hence a lot more concerted effort is needed by the Government, private organizations, religious and traditional leaders to educate and enlighten people on the need for protected sex, especially people with multiple sexual partners in order to reduce the spread of STIs including HIV and AIDS in our communities in Lagos, Nigeria.

\section{Social and behavioural aspects of prevention poster session 4: Health Services Venue Attendees}

\section{P2-S4.01 EPIDEMIOLOGICAL PROFILE AND MOTIVATIONS FOR HIV SCREENING AMONG PEOPLE ATTENDING AN ANONYMOUS HIV VOLUNTARY TESTING SERVICE IN COTONOU, BENIN}

doi:10.1136/sextrans-2011-050108.333

${ }^{1} \mathrm{D} \mathrm{M}$ Zannou, ${ }^{1} \mathrm{~A}$ Azon-Kouanou, ${ }^{1} \mathrm{O} \mathrm{M}$ Alaye, ${ }^{1} \mathrm{~J}$ Akakpo, ${ }^{2} \mathrm{D}$ Azon, ${ }^{2} \mathrm{E}$ Akinocho, ${ }^{3}$ S Anagonou-Y. ${ }^{1}$ Ambulatory treatment center of PLWHIV-CNHU, Cotonou, Benin; ${ }^{2}$ National Program to Fight against AIDS, Benin, Cotonou, Benin; ${ }^{3}$ Microbiology laboratory-CNHU-Cotonou, Cotonou, Benin
Background The creation of the only free anonymous HIV voluntary counselling and testing (VCT) center in Cotonou (named SIDAG) is an HIV prevention strategy that allows to reach those who decide, for themselves, to know their HIV status. Knowledge of the epidemiological profile and motivations of these volunteers will help tailoring services offered to them.

Objectives i) To describe practices at risk for HIV infection among the SIDAG's clientele ii) To analyse the motivations bringing these people for HIV screening; iii) To identify factors associated with HIV infection in this group. MethodsThis cross-sectional survey, carried out from April to June 2010, included all subjects attending SIDAG for HIV anonymous VCT who provided consent for participation. HIV testing was carried out immediately using an algorithm with two rapid tests (Determine and SD Bioline), and results were communicated on site to the participants before they leave.

Results 280 subjects participated in the study (149 men, 53\% and 131 women, 47\%). Their mean age was 28 years [range: 17-66 years]; $32 \%$ were college or university students, $53 \%$ had attained a higher educational level and $76 \%$ were single. $22 \%$ of the subjects had a history of STI. All subjects reported being heterosexual; 274 (98\%) had sexual partners (regular partners: $73 \%$; casual partners only: $25 \%$ ). $27 \%$ always used condoms for casual sex. $13 \%$ of the men reported sex with sex workers. The main motivations for getting tested were the desire to know their HIV status: $43.6 \%$; exposure to HIV through sexual contact: $20 \%$ (unprotected sex, condom failure, prolonged relation with HIV-infected partner); and peer pressure from family or friends: $11.2 \%$. The HIV serology was positive in $6.8 \%$ of the 280 tested subjects. Factors associated with seropositivity were: a low education level $(p<0.001)$, current or previous cohabitation with a sexual partner $(p<0.01)$, lack of condom use $(p<0.001)$ and motivation for testing because of clinical suspicion $(p<0.001)$.

Conclusion Candidates for anonymous HIV VCT in Cotonou are mainly young patients concerned by the risk behaviours they had Services like SIDAG should be decentralised to serve the greatest number.

\section{P2-S4.02 DON'T LOOK AT YOUR PATIENTS, LOOK AT THEIR PARTNERS: CHARACTERISTICS OF SEXUAL PARTNERSHIPS REPORTED BY PEOPLE ATTENDING GUM CLINICS IN ENGLAND}

doi:10.1136/sextrans-2011-050108.334

${ }^{1} \mathrm{C}$ H Mercer, ${ }^{1} \mathrm{C}$ R H Aicken, ${ }^{2} \mathrm{~N}$ Low, ${ }^{3} \mathrm{C}$ S Estcourt, ${ }^{4} \mathrm{P} \mathrm{J}$ White, ${ }^{5} \mathrm{~F}$ Keane, ${ }^{6} \mathrm{G}$ Brook, ${ }^{1} \mathrm{G}$ Rait, ${ }^{7} \mathrm{~J}$ A Cassell. ${ }^{1}$ University College London, London, UK; ${ }^{2}$ University of Bern, Bern, Switzerland; ${ }^{3}$ Barts and the London School of Medicine and Dentistry, London, UK; ${ }^{4}$ Health Protection Agency, London, UK; ${ }^{5}$ Royal Cornwall Hospitals NHS Trust, Truro, Cornwall, UK; ${ }^{6}$ Central Middlesex Hospital, London, UK; ${ }^{7}$ Brighton and Sussex Medical School, Brighton, UK

Background Partnership characteristics, for example, their length, timing, and whether or not condoms are used, may be more important for assessing STI transmission risk than partnership numbers. However, data routinely collected by GUM clinics in the UK are limited in terms of such measures of partnership risk. We sought to measure this in a high-risk population and consider the implications for STI transmission and partner notification (PN)

Methods Cross-sectional survey of 2203 people attending 4 sociodemographically and geographically contrasting GUM clinics in England in 2009. Attendees completed a questionnaire that was linked to their clinical records for data on acute STI diagnoses. Questions asked about their three most recent partnerships in the 3 months prior to attending GUM and the total number of partners in this period. We used a novel statistical approach to weight the data to represent the partnerships for which these questions were not asked. This enables us to describe the population of partnerships reported by GUM attendees, rather than the population of attendees. 


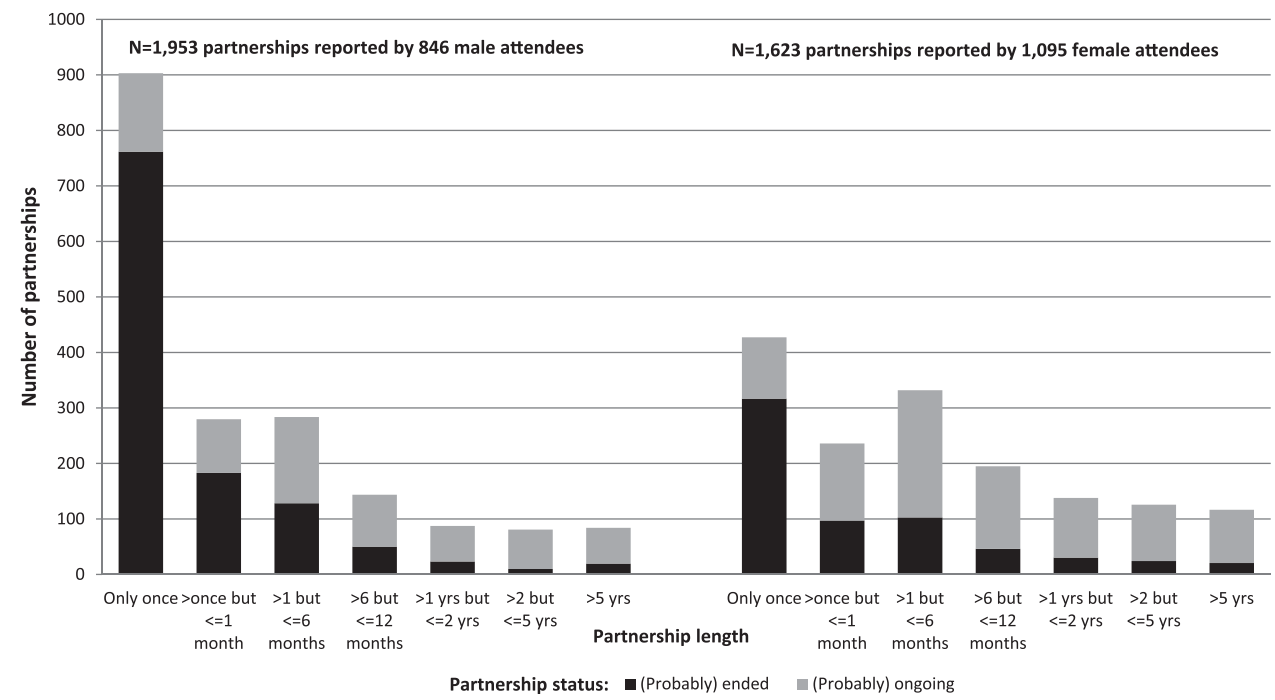

Abstract P2-S4.02 Figure 1 Distribution of partnership length by partnership status and gender of GUM clinic attendee.

Results Most patients had few partners: median: 1 partner, IORs: 1-3 (men) and 1-2 (women). However, the 1941 attendees reporting partners reported a total of 3576 partners: 1953 by 846 men, 1623 by 1095 women. Men's partnerships were shorter than women's: $73 \%$ vs $54 \%(p<0.01)$ were $<3$ months "old" (49\% vs $27 \%$, respectively, were one-off encounters), and were more likely to be considered as ended: $64 \%$ vs $41 \%$, respectively, $p<0.01$, see Abstract P2-S4.02 figure 1 Inconsistent condom use was high, especially in women's partnerships: $81 \%$ vs $65 \%$ among men's partnerships, $p=0.002$. Men (but not women) were more likely to have acute STIs diagnosed if they had never used condoms with their partners than if they had at least once: $33 \%$ vs $27 \%, p=0.001 .24 \%$ of men vs $14 \%$ of women had recent concurrent partnerships based on the dates of their 3 most recent partners $(p<0.01)$, but this was not associated with having acute STI diagnoses.

Conclusions Despite low median partner numbers, GUM attendees have a large population of partnerships, of which only a small minority involve consistent condom use, thus the potential for STI transmission is high. These partnerships are often casual in nature being short in duration and unlikely to be ongoing. Research is needed to develop and test alternative methods for reaching such partners to ensure that PN achieves both individual and public health benefit.

\section{P2-S4.03 PREVALENCE AND RISK FACTORS FOR CHLAMYDIA TRACHOMATIS INFECTION AMONG FEMALE MEDICAL STUDENTS AND WOMEN ATTENDING FAMILY PLANNING CLINICS IN NORTHERN ITALY}

doi:10.1136/sextrans-2011-050108.335

${ }^{1} \mathrm{~A}$ Biglino, ${ }^{1} \mathrm{G}$ Montrucchio, ${ }^{1} \mathrm{R}$ Moglia, ${ }^{1} \mathrm{E}$ Concialdi, ${ }^{1} \mathrm{C}$ Bolla, ${ }^{2} \mathrm{M}$ Tramontano, ${ }^{3} \mathrm{D}$ V Ferrero. ${ }^{1}$ "Cardinal Massaja" Hospital / University of Torino, Asti, Italy; ${ }^{2}$ Family Planning Clinic, A.S.L. 19, Asti, Italy: ${ }^{3}$ University of the Pacific, Stockton, USA

Background Prevalence and risk data concerning Chlamydia trachomatis CT in Italy is scare. We present a study of the prevalence and predictive risk factors for CT infection by comparing two different female populations at risk because of age (18-26) but with different socio-cultural backgrounds.

Methods An anonymous questionnaire concerning sexual behaviour was given to 493 female medical students aged 18-26 at the University of Torino and to women aged 18-26 attending three Family Planning Clinics (FPC) in Asti, Italy. A first-catch urine sample was collected into a liquid transport medium (UPT, Becton
Dickinson) from each subject who completed the questionnaire. Nucleic acid amplification testing for CT was performed using the $\mathrm{BD}$ ProbeTec ET assay. Age and behavioural characteristics were compared.

Results 6 women of 493 medical students tested (1.22\%) were positive for CT, while 11 of 262 women attending FPC had a positive CT result $(4.2 \%) \quad\left(\chi^{2}=5.7 ; p=0.017\right)$. Different behaviours between the female medical students and the FPC population respectively for non-barrier contraceptive methods were: (34\% vs $59 \%$ ); age $<18$ at first intercourse ( $40 \%$ vs $60 \%$ ); more than 1 partner during last year (16\% vs $20 \%$ ), or the last 5 years ( $2 \%$ vs $56 \%$ ). Risk of infection was associated with $>4$ partners during the last 5 years both in medical students and to a lesser extent in women attending FPC (OR 10.7; CI 2 to 49 and, respectively (OR 3.7; CI 1.2 to 12). Age at first intercourse was also a factor. Women in FPC whose age was $<18$ at first intercourse were at higher risk of CT (OR 7.6; CI 1.1 to 30) than the female medical students (OR 1.5; CI 0.3 to 8 ). Logistic regression confirmed that $>4$ partners during the last 5 years in both groups (Wald 7.3; $p<0.01$ [students]; 3.7 $\mathrm{p}<0.05$ [FPC subjects]) and an age $<18$ at first intercourse only in FPC subjects (Wald 3.5; $\mathrm{p}<0.06$ ) were independent predictors of infection.

Conclusions Female medical students showed a significantly lower prevalence of CT compared to the female population of same age but different socioeconomic background. The number of partners in the last 5 years and age at first intercourse were the factors regarding this difference. Awareness and the level of knowledge, possibly dependent on cultural factors, seem to reduce the prevalence. The importance of targeted CT screening programs to groups at greatest risk, while promoting widespread educational efforts and awareness of risk among general population is apparent.

\section{P2-S4.04 MOTIVATIONAL INTERVIEWING IS EFFECTIVE FOR REDUCING HIGH RISK SEXUAL BEHAVIOUR}

doi:10.1136/sextrans-2011-050108.336

${ }^{1} \mathrm{~J}$ Boman, ${ }^{2} \mathrm{H}$ Lindqvist, ${ }^{3} \mathrm{~A}$ Brandell-Eklund, ${ }^{2} \mathrm{~L}$ Forsberg, ${ }^{1} \mathrm{E}$ Nylander. ${ }^{1}$ Umeå University, Umeå, Sweden; ${ }^{2}$ Karolinska Institute, Sweden; ${ }^{3}$ Swedish National Institute of Public Health, Sweden

Background Motivational interviewing (MI) can be effective for behaviour change even when tailored to brief encounters. MI is focused on resolving the ambivalence that might prevent change. 